\title{
Tell me how to teach, I'll learn how to solve problems
}

\author{
Noboru Matsuda ${ }^{1}$, Nikolaos Barbalios ${ }^{2}$, Zhengzheng Zhao ${ }^{3}$, Anya Ramamurthy ${ }^{4}$, \\ Gabriel J. Stylianides ${ }^{5}$, and Kenneth R. Koedinger ${ }^{6}$ \\ ${ }^{1,2,3}$ College of Education and Human Development, Texas A\&M University \\ ${ }^{1}$ Noboru.Matsuda@tamu.edu, ${ }^{2}$ nbarmpalios@gmail.com, ${ }^{3}$ zhengzhaoap@tamu.edu \\ ${ }^{4,6}$ Human-Computer Interaction Institute, Carnegie Mellon University \\ ${ }^{4}$ anya.ramamurthy@gmail.com, ${ }^{6}$ koedinger@cs.cmu.edu \\ ${ }^{5}$ Department of Education, University of Oxford \\ 5abriel.stylianides@education.ox.ac.uk
}

\begin{abstract}
In this paper we study the effect of adaptive scaffolding to learning by teaching. We hypothesize that learning by teaching is facilitated if (1) students receive adaptive scaffolding on how to teach and how to prepare for teaching (the metacognitive hypothesis), (2) students receive adaptive scaffolding on how to solve problems (the cognitive hypothesis), or (3) both (the hybrid hypothesis). We conducted a classroom study to test these hypotheses in the context of learning to solve equations by teaching a synthetic peer, SimStudent. The results show that the metacognitive scaffolding facilitated tutor learning (regardless of the presence of the cognitive scaffolding), whereas cognitive scaffolding had virtually no effect. The same pattern was confirmed by two additional datasets collected from two previous school studies we conducted.
\end{abstract}

Keywords: Teachable Agent, Learning by Teaching, Algebra, Adaptive Scaffolding, SimStudent.

\section{Introduction}

Learning by teaching [1] is known to be effective with empirical evidence of students learning by teaching their peers in various domains [2], across different student populations [3], with different types of interactions and formats of tutoring [4]. In this paper, we use the term tutor learning to refer to the effect of learning by teaching on the tutor (i.e., a student who teaches his/her peer) [5]. For the currently study, our focus is on tutor learning in the mathematical domain of solving linear equations.

There has been growing interest in the application of teachable agents to study the effect of tutor learning, in particular in the field of artificial intelligence in education and human-computer interaction [6-8]. Teachable agents are synthetic peers that students can interactively teach.

Using the teachable agent technology, researchers try to understand the effect of tutor learning by, for example, mining stereotypic patterns of effective tutoring interactions [9], analyzing cognitive factors that contribute to tutor learning [8], and studying student's perceptions and motivations while interacting with synthetic peers [10]. Yet, the underlying cognitive mechanism of tutor learning is not fully understood. Without clear understanding of what makes learning by teaching effective, it is 
impractical to build a technology to facilitate tutor learning despite its promising potential for efficacy and large-scale dissemination.

Learning by teaching is a complicated phenomenon with many factors to be explored. As part of our on-going effort to contribute to advancing cognitive and social theory of tutor learning, the goal of the current paper is to study the effect of adaptive scaffolding to facilitate tutor learning, which is motivated by our past study findings as described in the next section.

\section{$2 \quad$ Learning by Teaching: Lessons Learned}

To understand how and why students learn by teaching others, we have developed an online learning environment for learning to solve equations by teaching, called APLUS (described in section 4). In APLUS, students learn by teaching a synthetic peer, called SimStudent [8]. Prior to the current study, APLUS has been used in five Algebra classroom studies with more than 1,000 middle school students.

Throughout these classroom studies, we have addressed a number of research questions such as questions about the effect of answering tutee's questions [11] and the effect of extrinsic motivation for tutor learning [12].

One of the most important findings thus far is that learning by teaching may not be effective when students do not have sufficient prior knowledge on the task (how to solve equations in our case) and do not know how to teach properly [8]. In previous studies, we often observed that students taught their synthetic peers incorrectly without realizing they were making mistakes. Students also often taught their peers inappropriately - e.g., only teaching "easy" problems, causing the synthetic peer to fail to develop sufficient skills to solve a wide range of equations.

\section{$3 \quad$ Research question and hypothesis}

Our previous studies strongly suggest that students need an assistance for successful teaching in order to facilitate tutor learning. We then hypothesize that providing adaptive scaffolding will resolve this issue. What kinds of scaffolding should be provided? From the past studies, we have two working hypotheses.

First, students need to correctly teach their peers how to solve problems. However, due to the lack of sufficient prior knowledge, students often make mistakes and get stuck. Adaptive scaffolding on how to solve problems is therefore necessary-we call this the cognitive scaffolding. We hypothesize that adaptive cognitive scaffolding will be particularly important for students with low prior knowledge since some level of knowledge is necessary for students to have in order to be able to teach their synthetic peers- the cognitive scaffolding hypothesis.

Second, students need to know how to teach their peers appropriately. Students need to know, for example, what problem might be useful to teach next and when to quiz their peers. Adaptive scaffolding on how to teach is therefore necessary-we call this the metacognitive scaffolding. Even with a low prior knowledge, students might recognize their mistakes and acquire correct skills on how to solve problems while teaching if appropriate feedback is given from the tutoring interaction-e.g., the 
summary of a formative assessment reveals an inconsistency between the student's belief and actual correctness. However, to receive effective feedback, students must teach their peers properly. Therefore, adaptive metacognitive scaffolding is essential- the metacognitive scaffolding hypothesis.

Third, it might be the case that students need both cognitive and metacognitive scaffolding for successful learning by teaching - the hybrid scaffolding hypothesis.

Our research question centers on which of these types of adaptive scaffolding facilitate tutor learning. To test our hypotheses, we implemented the cognitive and metacognitive scaffolding on APLUS (section 4) and conducted a classroom study with the extended APLUS (section 5).

\section{Technology Innovation for Learning by Teaching}

We have developed an online environment called APLUS (Artificial Peer Learning environment Using SimStudent) where students learn to solve algebra equations by teaching a synthetic peer, SimStudent.

SimStudent is a machine learning agent that interactively learns cognitive skills in the form of production rules through guided-problem solving [13]. SimStudent is an implementation of programming by demonstration in the form of inductive logic programming. This is made possible by generalizing examples that show when to apply particular skills. In the context of learning by teaching, feedback and hints on a step provided by the student to SimStudent become examples.

Fig. 1 shows an example screenshot of APLUS. Details of APLUS have been published elsewhere (for example [8]); hence we only provide a brief explanation here. SimStudent is shown as an avatar on the bottom left corner. To teach SimStudent how to solve equations, a student must enter a problem into the tutoring interface (' $a$ ' in Fig. 1). SimStudent will then attempt to solve the problem one step at a time by applying the skills learned so far. On each step, if SimStudent can make a suggestion, the student is prompted to provide yes/no feedback about the correctness of the suggested step ('b' in Fig. 1). Positive feedback ("yes") indicates that the student agrees that the step SimStudent suggested is correct, in which case SimStudent proceeds to the next step. Negative feedback ("no") indicates the student's disagreement. When given negative feedback, SimStudent attempts to apply another skill and make another suggestion. If SimStudent cannot find a skill to apply, SimStudent asks the student to demonstrate the next step, and the student then performs the actual step on the tutoring interface.

When a student provides negative feedback to a step SimStudent suggested, SimStudent occasionally asks the student to explain why he/she thinks that the step is wrong [11]. Fig. 2 shows an example screenshot of SimStudent asking a "why" question. SimStudent can also compare (1) a previous step in which the same skill was applied and received positive feedback and (2) the current step which just received negative feedback. SimStudent then asks the student why the current step is incorrect. To proceed with the tutoring process, the student must answer SimStudent's question in a free-form text. 


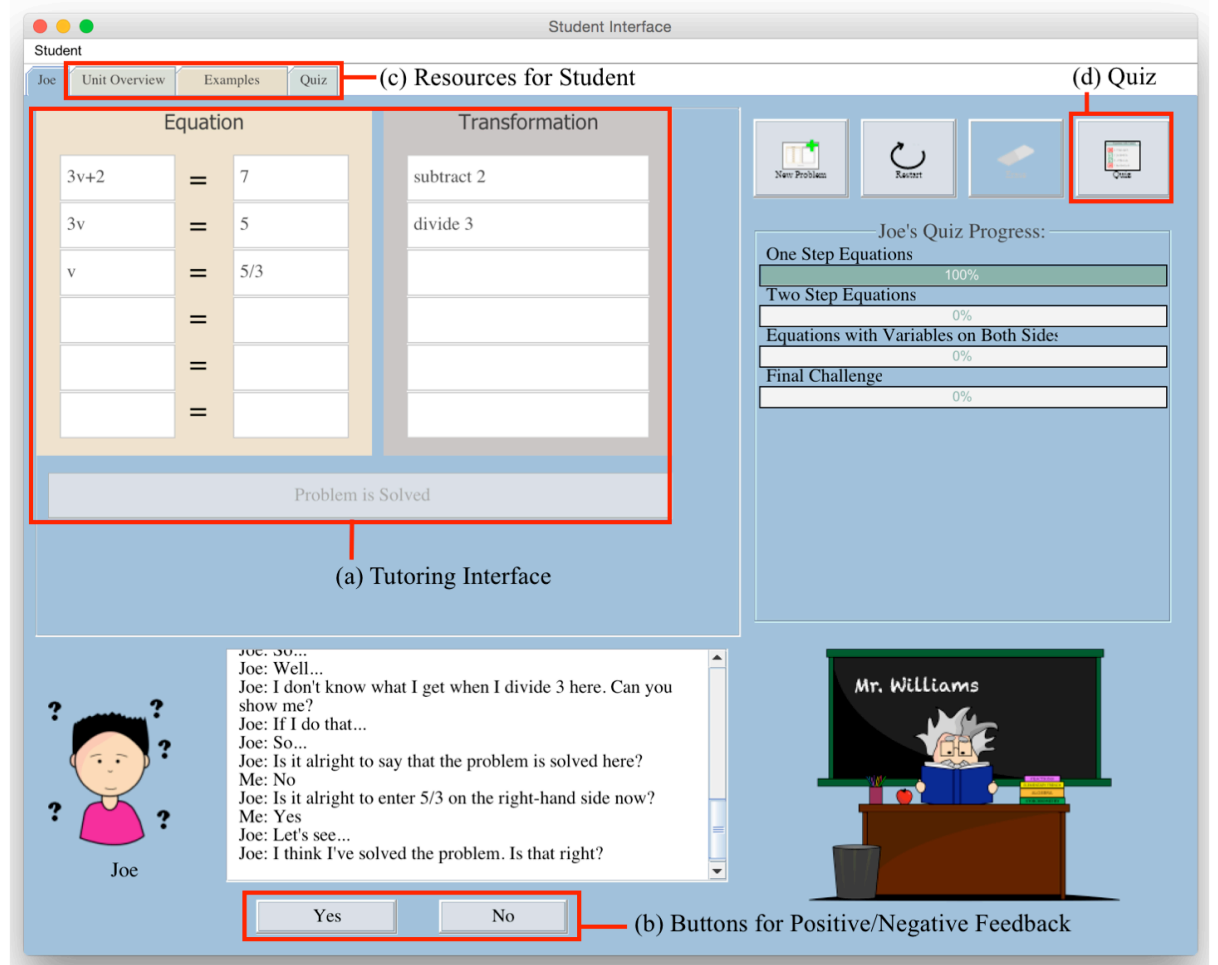

Fig. 1. An example screenshot of APLUS.

Students' goal is to have their SimStudent pass the quiz ('c' in Fig. 1). When SimStudent is quizzed by a student, it attempts to solve quiz problems in a target section by applying already learned skills. A teacher agent called Mr. Williams, as shown on the lower right corner in Fig. 1, then provides a summary of the quiz results, and the student can review the exact solutions made by SimStudent one by one. APLUS also includes resources for students to review ('d' in Fig. 1) and prepare for tutoring. The resources include worked-out examples with brief explanations on solutions and a unit overview that provides a quick introduction to equation solving.

We have recently modified Mr. Williams to provide adaptive scaffolding in two ways: (1) Cognitive scaffolding provides adaptive assistance on how to solve equations. When a student is not sure about the correctness of a step SimStudent performed, or when they do not know what a correct next step is, he/she can click on Mr. Williams to ask for help. Mr. Williams then provide a just-in-time, contextualized assistance to overcome the student's impasse. (2) Metacognitive scaffolding provides adaptive assistance on how to teach SimStudent. When a student is not sure about how to proceed tutoring, he/she can click on Mr. Williams to ask for help. Four types of assistance are provided: (a) the quiz assistance suggests when students should take the quiz and explains why, (b) the problem selection assistance suggests what problem students should pose next and explains why, (c) the resource assistance suggests when students should review a particular resource and why, and (d) the impasse re- 


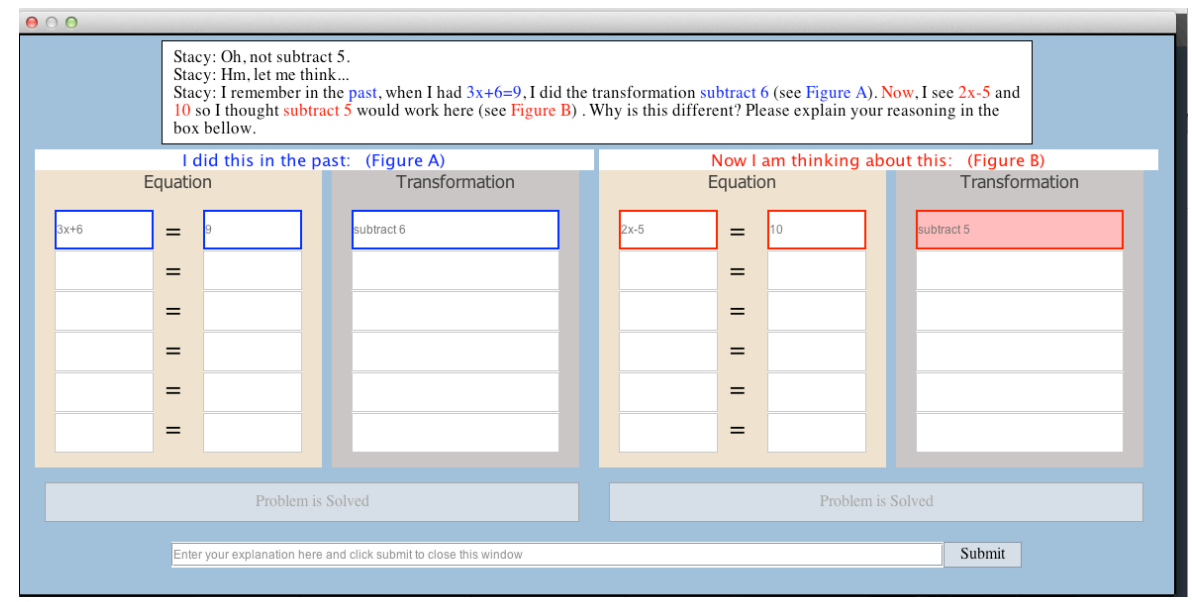

Fig. 2. SimStudent is asking student to explain why the step is wrong.

covery assistance suggests students should restart a problem or give a new problem when they are stuck for a long-enough time.

\section{Evaluation Study}

To test the hypotheses on the effectiveness of adaptive scaffolding as mentioned in the previous section, we conducted a classroom in-vivo study in Algebra classes at three urban public middle schools in the greater Pittsburgh area in Pennsylvania.

\subsection{Method}

The study was a randomized controlled trial with three conditions. (1) The metacognitive condition (MC for short) used a version of APLUS with metacognitive scaffolding only. (2) The cognitive condition (C for short) used APLUS with cognitive scaffolding only. (3) The hybrid condition (MC+C for short) used APLUS with both metacognitive and cognitive scaffolding.

In total, 364 students (7th and 8th grade) participated in the study from 22 algebra classes. Students were randomly assigned to one of the three conditions.

The study lasted for six consecutive days with one classroom period (42 minutes each) per day. On the first day, all students took an online pre-test. On the second day, students first watched a 6-minute introduction video on how to use APLUS, and then started tutoring SimStudent. Students used APLUS for four days. On the sixth day, students took an online post-test.

\subsection{Measures}

Students' learning outcome was measured with the online test scores. The online test consisted of two parts: a Procedural Skill Test and a Conceptual Knowledge Test.

The Procedural Skill Test has three sections: (a) an equation section that contains 10 problems; 2 one-step equations, 2 two-step equations and 6 equations with variables on both sides; (b) an effective next step section that has 2 equation problems, 
each showing an intermediate solution step with four candidates for a next step and asking students to indicate if each candidate is correct or not; and (c) an error detection section with 3 equation problems, each showing an incorrect solution for which students are asked to identify the incorrect step and explain their reasoning.

The Conceptual Knowledge Test consists of 24 true/false multiple choice questions, with 7 items asking about variable terms, 6 asking about constant terms, 6 asking about like terms, and 5 asking about equivalent terms.

In addition to the learning outcome data, we also used the process data that are detailed interactions between student and system that APLUS automatically logs (e.g., problems used for tutoring, tutored steps, quiz frequency, etc.).

\subsection{Results}

For the following analysis, we included only those students who took both the preand post-tests, and "completed" teaching, which we define as either the student participated in all four days of teaching SimStudent or had SimStudent pass all quiz sections. As a result, 257 students are included in the analysis below- -89 in the $\mathrm{C}$ condition, 88 in the $\mathrm{MC}$ condition, and 80 in the $\mathrm{MC}+\mathrm{C}$ condition.

\subsubsection{Test Scores}

Table 1 shows scores for Conceptual Knowledge Test (CKT) and Procedural Skill Test (PST). There is no condition difference $(\mathrm{C}$ vs. $\mathrm{MC}$ vs. $\mathrm{MC}+\mathrm{C})$ in pre-test score both for $\operatorname{CKT}(\mathrm{F}(2,254)=0.17, p=0.85)$ and $\operatorname{PST}(\mathrm{F}(2,254)=1.21, p=0.30)$. We then ran a repeated-measures ANOVA, for CKT and PST separately, with test scores as a dependent variable, and test-time (pre vs. post) and condition (C vs. MC vs. $\mathrm{MC}+\mathrm{C}$ ) as independent variables.

Table 1. Test scores both for CKT and PST.

\begin{tabular}{llllll}
\hline & \multicolumn{2}{c}{ CKT } & & \multicolumn{2}{c}{ PST } \\
\cline { 2 - 3 } \cline { 5 - 6 } & Pre-test & Post-test & & Pre-test & Post-test \\
\hline MC+C & $.42(.24)$ & $.48(.20)$ & & $.54(.22)$ & $.63(.24)$ \\
MC & $.40(.24)$ & $.50(.21)$ & & $.53(.23)$ & $.62(.23)$ \\
$\mathbf{C}$ & $.41(.24)$ & $.46(.20)$ & & $.49(.21)$ & $.54(.23)$ \\
\hline
\end{tabular}

For the conceptual test (CKT), there is a main effect of test-time $\left(M_{\text {Pre }}=0.41 \pm 0.24\right.$ vs. $\left.M_{\text {Post }}=0.48 \pm 0.20 ; \mathrm{F}(1,254)=22.52, p<0.001, d=0.28\right)$, but there is no main effect of condition $\left(M_{C}=0.44 \pm 0.22\right.$ vs. $M_{M C}=0.45 \pm 0.23$ vs. $M_{M C+\mathrm{C}}=0.46 \pm 0.22 ; \mathrm{F}(2$, $254)=0.26, p=0.77)$. The current version of APLUS enhanced students' understanding of algebra concepts measured in CKT. The type of adaptive scaffolding does not have any impact on students' learning on conceptual knowledge measures in CKT.

For the procedural test (PST), the repeated-measures ANOVA suggested the existence of an interaction between test-time and condition; $\mathrm{F}(2,254)=2.85, p=0.06$. A simple main effect on condition (paired t-test with test-time as the independent variable) revealed that students in all conditions showed a reliable increase in PST test scores, but the effect size is notably smaller in $\mathrm{C}$ condition; $\mathrm{C}$ : paired-t $(88)=-2.55, p$ $=0.01, \boldsymbol{d}=\mathbf{0 . 2 0} ;$ MC: paired-t $(87)=-5.07, p<0.001, \boldsymbol{d}=\mathbf{0 . 4 1} ; \mathrm{MC}+\mathrm{C}$ : paired-t(79) 
$=-5.54, p<0.001, \boldsymbol{d}=\mathbf{0 . 4 0}$. A simple main effect analysis on PST post-test (an ANOVA with condition as the independent variable) revealed condition as a main effect; $F(2,253)=3.95, p<0.05$. The post-hoc tests confirmed that both MC and $\mathrm{MC}+\mathrm{C}$ students scored reliably higher on the PST post-test than $\mathrm{C}$ students $(t(175.0)$ $=2.41, p<0.05$ for $\mathrm{MC}$ and $t(163.5)=2.64, p<0.01$ for $\mathrm{MC}+\mathrm{C})$, but there is no reliable difference between $\mathrm{MC}$ and $\mathrm{MC}+\mathrm{C}$ students; $t(161.9)=-0.37, p=0.71$.

In sum, metacognitive scaffolding is helpful but cognitive scaffolding does not appear to amplify the effect of tutor learning. In other words, adding cognitive scaffolding to metacognitive scaffolding does not yield better effects than metacognitive scaffolding alone.

Since the current study does not include a baseline condition where no scaffolding is available, we compared the current study with two previous studies we conducted: Study IV [14] where metacognitive scaffolding (MC) was compared with no scaffolding (BL), and Study $\mathrm{V}$ where metacognitive plus cognitive scaffolding $(\mathrm{MC}+\mathrm{C})$ was compared with no scaffolding (BL). In Study IV there were 173 students (7th through 9th grade) in nine Algebra-I classes, whereas in Study V there were 318 students (7th and 8th grades) in 14 Pre-Algebra and 3 Algebra I classes from two schools.

Fig. 3 shows a bar graph of PST pre- and post-test scores. In the graph, the current study is denoted as Study VI. The relative high test scores in Study IV is arguably due to the population difference (the only study with 9 th graders).

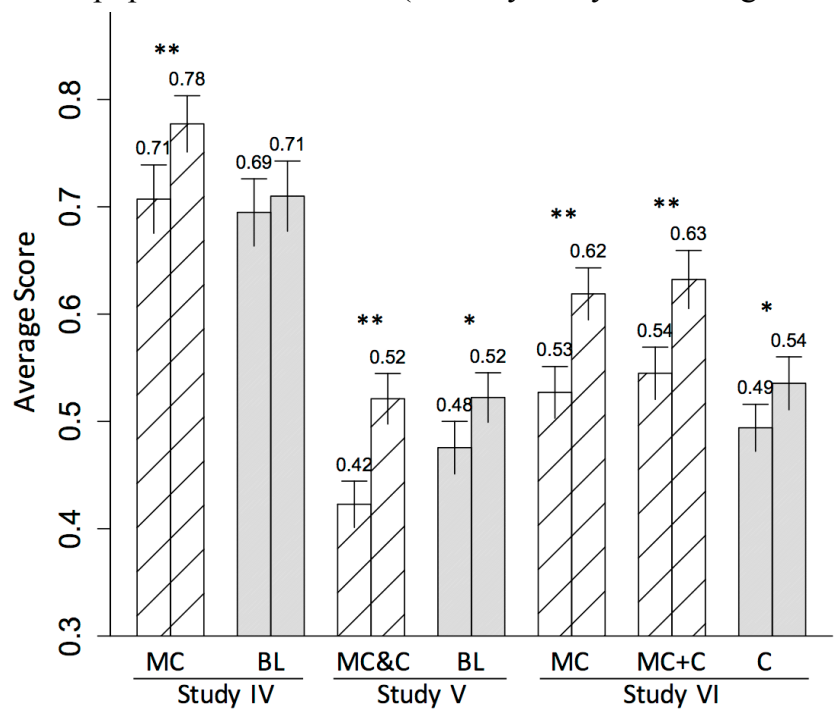

Fig. 3. PST scores for 7 conditions in Study IV, V, and the current study (Study VI). ** $\mathrm{p}<0.01, * \mathrm{p}<0.05$
We tested two hypotheses: (C1) all four conditions with metacognitive scaffolding, regardless of the availability of cognitive scaffolding, show the same gain from pre- to posttests; and (C2) the $\mathrm{C}$ condition in Study VI shows the same gain from pre- to post-test as two BL conditions in Study IV and V.

To test these two hypotheses, we ran two repeated-measures

ANOVAs, one for each hypothesis. For (C1), there is a main effect of

test-time; $\mathrm{F}(1,319)=100.42, \mathrm{p}<0.001$. There is also a main effect of condition; $\mathrm{F}(3$, $319)=19.29, \mathrm{p}<0.001$. There is no statistically reliable interaction between testtime and condition. The same pattern is found for $(\mathrm{C} 2)$ : a main effect of test-time $(\mathrm{F}(1,245)=11.77, \mathrm{p}<0.001)$; condition $(\mathrm{F}(2,245)=18.42, \mathrm{p}<0.001)$; and no interactions between them. 
The data collected from three independent classroom studies all suggest that metacognitive scaffolding facilitates tutor learning, regardless of the availability of cognitive scaffolding. However, cognitive scaffolding does not necessarily facilitate tutor learning (or is equally "effective" as the baseline), and adding cognitive scaffolding to metacognitive scaffolding is as effective as the metacognitive scaffolding alone.

\subsubsection{Effect of cognitive scaffolding and students' prior knowledge}

To see if cognitive scaffolding helped certain students, we categorized students in the cognitive scaffolding condition into four groups based on their prior knowledge as measured in the pre-test score. Table 2 shows procedural pre- and post-test (PST) scores. The quartile Q1 represents the students who scored lowest on the pre-test.

Table 2. Procedural pre- and post-test (PST) scores for students in the cognitive scaffolding condition. Students are grouped based on their pre-test quartile.

\begin{tabular}{|c|c|c|c|c|}
\hline & Q1 & Q2 & Q3 & Q4 \\
\hline Pre & $0.37(0.16)$ & $0.40(0.13)$ & $0.54(0.17)$ & $0.66(0.21)$ \\
\hline Post & $0.44(0.16)$ & $0.47(0.24)$ & $0.53(0.25)$ & $0.70(0.18)$ \\
\hline
\end{tabular}

A repeated-measures ANOVA with test score as a dependent variable and test-time (pre vs. post) and quartile (Q1 Q4) as independent variables revealed a main effect for quartile; $\mathrm{F}(3,85)=11.77, p<0.001$. Test-time is also a main effect; $\mathrm{F}(1,85)=$ $6.57, p=0.01$. There is no statistically reliable interaction between test-time and quartile. This result suggests that the "effect" of cognitive scaffolding does not change by students' prior knowledge.

\subsubsection{Why is the metacognitive scaffolding effective?}

We have yet to fully understand what makes metacognitive scaffolding effective. So far, we found the following. First, the effectiveness of metacognitive scaffolding does not change based on the student's prior knowledge (measured as pre-test score). This is confirmed by dividing students into quartiles based on their PST pre-test scores.

Second, in a previous study, the data suggested that metacognitive scaffolding on problem selection (i.e., what problem should be taught next) actually influenced students to pose more appropriate problems to SimStudent, which in turn facilitated tutor learning [14]. However, the effect of metacognitive scaffolding on problem selection is not confirmed in the current study.

Third, there is no notable correlation between the number of metacognitive hints received and PST post-test scores when pre-test score is controlled. A stepwise regression revealed that the number of quiz hints received is a statistically reliable predictor of post-test score $(\mathrm{F}(1,160)=6.19, p=0.01)$. However, students quizzed SimStudent an average of nine times in all three conditions.

\section{Discussion}

The metacognitive hypothesis has been supported. The current and past two classroom studies all show that metacognitive scaffolding (helping students to teach and prepare for teaching) is an essential component for successful learning by teaching, whereas cognitive scaffolding (helping students to solve equations) has no effect rela- 
tive to no scaffolding. In the current implementation, the metacognitive scaffolding is operationalized to support students' understanding of how to select appropriate problems to teach, when to quiz their peers, and when to review study materials to prepare themselves for teaching (e.g., reviewing worked-out examples and unit overview).

We have yet to fully understand why metacognitive scaffolding, as we defined it in this paper, helps. One hypothesis is that understanding (and actually applying) proper teaching strategies increases the likelihood for students to be exposed to opportunities to learn correct skills (e.g., from worked-out examples) and also to face the knowledge gap (e.g., a step that a student believes to be correct is marked as incorrect on the quiz summary). If these ideas are actually the key events that drive tutor learning, then guiding students to these key events should facilitate students' learning.

Further research is necessary to understand the underlying mechanism of tutor learning. We are currently analyzing the process data showing detailed interaction between students and SimStudent. Sequence mining is one potential technique to address the question of why metacognitive scaffolding helps.

We were surprised that the current data do not provide evidence that cognitive scaffolding, as we defined it in this paper, helps tutor learning. It might be the case, however, that the current implementation of cognitive scaffolding needs to be improved. Students might have used the cognitive scaffolding as a mere mechanism to provide correct feedback and hint-similar to those students excessively asking for hints when using cognitive tutors just to perform a step correctly-that must be discouraged. A future study will be designed to explore this new hypothesis.

\section{Conclusion}

We found that the adaptive scaffolding on how to tutor and how to prepare for tutoring (the metacognitive scaffolding) facilitates tutor learning, while the adaptive scaffolding on how to solve problems (the cognitive scaffolding) has virtually no impact on tutor learning. In the present study, the metacognitive scaffolding provided just-intime assistance on what problem should be taught next, when to quiz (i.e., a formative assessment), when to review resources to prepare for tutoring, and when to recover from an impasse. The cognitive scaffolding provided assistance on the correctness of the steps performed by the peer (to provide feedback to the peer), and the next step to be performed (to provide hints to the peer on what to do next).

In our classroom studies, we often see students get excited about interactively teaching on a computer with actual dialogue with a synthetic agent. Our data from recent classroom studies consistently show evidence of the effect of learning by teaching. Understanding how and why metacognitive scaffolding helps but not cognitive scaffolding is therefore an important research agenda to further advance the theory of learning by teaching and to build an effective technology for learning by teaching.

\section{Acknowledgement}

The research reported here was supported by National Science Foundation Award No. DRL-1252440 


\section{References:}

1. Gartner, A., M. Kohler, and F. Riessman, Children teach children: Learning by teaching. 1971, New York, NY: Harper \& Row.

2. Cohen, P.A., J.A. Kulik, and C.l.C. Kulik, Education outcomes of tutoring: A meta-analysis of findings. American Educational Research Journal, 1982. 19(2): p. 237-248.

3. Robinson, D., J. Schofield, and K. Steers-Wentzell, Peer and Cross-Age Tutoring in Math:Outcomes and Their Design Implications. Educational Psychology Review, 2005. 17(4): p. 327-362.

4. Cohen, E.G., Restructuring the classroom: Conditions for productive small groups. Review of Educational Research, 1994. 64(1): p. 1-35.

5. Roscoe, R.D. and M.T.H. Chi, Understanding tutor learning: Knowledgebuilding and knowledge-telling in peer tutors' explanations and questions. Review of Educational Research, 2007. 77(4): p. 534-574.

6. Biswas, G., et al., Learning by teaching: a new agent paradigm for educational software. Journal Applied Artificial Intelligence, 2005. 19(3\&4): p. 363-392.

7. Bredeweg, B., et al., DynaLearn - Engaging and Informed Tools for Learning Conceptual System Knowledge, in Cognitive and Metacognitive Educational Systems (MCES2009), AAAI Fall Symposium, P. R., R. Azevedo, and G. Biswas, Editors. 2009, AAAI Press: Arlington, VA. p. 46-51.

8. Matsuda, N., et al., Cognitive anatomy of tutor learning: Lessons learned with SimStudent. Journal of Educational Psychology, 2013. 105(4): p. 1152-1163.

9. Biswas, G., et al., Measuring Self-Regulated Learning Skills through Social Interactions in a teachable Agent Environment. Research and Practice in Technology Enhanced Learning, 2010: p. 123-152.

10. Okita, S.Y., J. Bailenson, and D.L. Schwartz, The mere belief of social interaction improves learning, in The Proceedings of the 29th Meeting of the Cognitive Science Society, D.S. McNamara and J.G. Trafton, Editors. 2007: Nashville. p. 1355-1360.

11. Matsuda, N., et al., Studying the Effect of Tutor Learning using a Teachable Agent that asks the Student Tutor for Explanations, in Proceedings of the International Conference on Digital Game and Intelligent Toy Enhanced Learning (DIGITEL 2012), M. Sugimoto, et al., Editors. 2012, IEEE Computer Society: Los Alamitos, CA. p. 25-32.

12. Matsuda, N., et al., Motivational factors for learning by teaching: The effect of a competitive game show in a virtual peer-learning environment, in Proceedings of International Conference on Intelligent Tutoring Systems, S. Cerri and W. Clancey, Editors. 2012, Springer-Verlag: Heidelberg, Berlin. p. 101-111.

13. Matsuda, N., W.W. Cohen, and K.R. Koedinger, Teaching the Teacher: Tutoring SimStudent leads to more Effective Cognitive Tutor Authoring. International Journal of Artificial Intelligence in Education, 2015. 25: p. 1-34.

14. Matsuda, N., et al., Investigating the Effect of Meta-Cognitive Scaffolding for Learning by Teaching, in Proceedings of the International Conference on Intelligent Tutoring Systems, S. Trausen-Matu, et al., Editors. 2014, Springer: Switzerland. p. 104-113. 